\title{
MicroRNA-122 is involved in oxidative stress in isoniazid-induced liver injury in mice
}

\author{
L. Song ${ }^{1,2}$, Z.R. Zhang ${ }^{1}$, J.L. Zhang ${ }^{1}$, X.B. Zhu' ${ }^{1}$, L. He ${ }^{1}$, Z. Shi' ${ }^{1}$ L. Gao', Y. Li', \\ B. Hu${ }^{1}$ and F.M. Feng ${ }^{1}$ \\ ${ }^{1}$ Hebei Province Key Laboratory of Occupational Health and Safety for Coal Industry, \\ School of Public Health, North China University of Science and Technology, \\ Tangshan, China \\ ${ }^{2}$ Center for Disease control and Prevention of Bayannur, Bayannur, China \\ Corresponding author: F.M. Feng \\ E-mail: fm_feng@sina.com \\ Genet. Mol. Res. 14 (4): 13258-13265 (2015) \\ Received May 6, 2015 \\ Accepted July 24, 2015 \\ Published October 26, 2015 \\ DOI http://dx.doi.org/10.4238/2015.October.26.22
}

\begin{abstract}
Many studies have shown that the pathogenesis of liver injury includes oxidative stress. MicroRNA-122 may be a marker for the early diagnosis of drug-induced liver injury. However, the relationship between microRNA-122 and oxidative stress in anti-tuberculosis druginduced liver injury remains unknown. We measured changes in tissue microRNA-122 levels and indices of oxidative stress during liver injury in mice after administration of isoniazid, a first-line anti-tuberculosis drug. We quantified microRNA-122 expression and indices of oxidative stress at 7 time points, including 1, 3, and 5 days and 1, 2, 3, and 4 weeks. The tissue microRNA-122 levels and oxidative stress significantly changed at 3 and 5 days, suggesting that isoniazid-induced liver injury reduces oxidative stress and microRNA-122 expression compared to in the control group $(P<0.05)$. Notably, over the time course of isoniazid-induced liver injury, mitochondrial ribosome protein S11 gene, the target of microRNA-122, began to change at 5 days $(P<0.05)$. The tissue microRNA-122 profile may affect oxidative stress by regulating mitochondrial ribosome protein
\end{abstract}


S11 gene during isoniazid-induced liver injury, which may contribute to the response mechanisms of microRNA-122 and oxidative stress.

Key words: Anti-tuberculosis drug-induced liver injury; Isoniazid; MicroRNA-122; Mitochondrion; Oxidative stress

\section{INTRODUCTION}

MicroRNAs (miRNAs) are short (18-25 nucleotides) endogenous, non-coding RNA molecules that regulate gene expression (Henderson and Azorsa, 2012). Studies have revealed unexpected diversity in their biogenesis pathways and regulatory mechanisms (Aghabozorg Afjeh and Ghaderian, 2013; Fitzgerald and Caffrey, 2014). Among miRNAs, microRNA-122 (miR-122), which accounts for approximately $70 \%$ of the total miRNA in the adult liver, are involved in liver biology and disease, including cell cycle progression, hepatocellular carcinogenesis (Wang et al., 2012), lipid metabolism (Tsai et al., 2012), and fibrosis (Arataki et al., 2013). Moreover, miR-122 is a potential biomarker for diagnosing hepatotoxicity caused by acetaminophen (Antoine et al., 2013), alcohol (Zhang et al., 2010), and drug-induced liver injury. Additionally, miR-122 can be used as a biomarker for anti-tuberculosis drug-induced liver injury (ADLI).

ADLI, a serious adverse effect of tuberculosis, which remains a global scourge treatment, may negatively affect treatment compliance. Isoniazid (INH) is used as a first-line drug to treat tuberculosis; however, ADLI is the most frequent side effect of INH and progresses into liver cirrhosis. ADLI occurs because of excessive oxidative stress and mitochondria dysfunction caused by reactive metabolites formed during drug metabolism (Zhang et al., 2011).

Oxidative stress refers to the high production of active oxygen free radicals, known as reactive oxygen species (ROS), and reactive nitrogen radicals, known as reactive nitrogen species. The cell cannot scavenge such high levels, creating an imbalance when cells use oxygen to produce ATP, leading to cell and tissue damage. ROS, an oxidative stress marker, is generated in and removed via the mitochondrion (Shahraki et al., 2013).

Recent studies have shown that mitochondrial ribosomal protein S11 (MRPS11) is targeted and regulated by miR-122 (Burchard et al., 2010; Liu et al., 2014). It is well known that oxidative stress causes ADLI. Although much is known about the effect of liver injury, information regarding the role of miR-122 in oxidative stress during ADLI is limited.

We hypothesized that certain types of miR-122, acting as a potential biomarkers for ADLI, regulate the synthesis of mitochondrial, thus participating in oxidative stress. We analyzed the expression of miR-122 and relative oxidative stress indicators over time in mice with INH-induced ADLI to determine whether miR-122 is involved in oxidative stress.

\section{MATERIAL AND METHODS}

\section{Ethics statements}

This study was carried out in strict accordance with the recommendations in the Guide for the Care and Use of Laboratory Animals of the National Institutes of Health. The protocol was approved by the Committee on the Ethics of Animal Experiments of the Hebei United University (Permit No.: 2012-20). 


\section{Animal experiments}

A total of 80 5-week-old Kunming mice (40 males and 40 females; body weight 18-22 g; Certificate No.: 2009-0004) were obtained from Beijing HFK Bioscience Co., Ltd. (Beijing, China). To establish the ADLI models, mice were administered $0.2 \mathrm{~mL} \mathrm{INH}$ orally at $90 \mathrm{mg} / \mathrm{kg}$ body weight (Shenyang Pharmaceutical University, Liaoning, China) dissolved in double-distilled water. Blood and liver tissue samples were collected at 1,3 and 5 days and 1,2, 3, and 4 weeks after administering $\mathrm{INH}(\mathrm{N}=10$ per time point/treatment group) and double-distilled water $(\mathrm{N}=$ 10 per time point/control group). Blood and liver tissue samples were centrifuged and the serum was collected and stored at $-80^{\circ} \mathrm{C}$ until use. A part of the liver from the porta was fixed in buffered neutral $10 \%$ formalin, and the remnant liver was stored at $-80^{\circ} \mathrm{C}$.

\section{Quantitative reverse transcription-polymerase chain reaction (PCR)}

Total RNA was isolated from mouse livers using a bench-top homogenizer with TRIzol Reagent (Invitrogen, Carlsbad, CA, USA) according to the manufacturer protocol. Total RNA concentration was quantified using a spectrophotometer (TU-1901; Beijing Purkinje General Instrument Co., Ltd., Beijing, China). The extracted RNA was tested for quality based on the RNA 28S/18S ratio and RNA integrity. Following the instructions of the TaqMan MicroRNA Reverse Transcription Kit (Applied Biosystems, Foster City, CA, USA), complementary DNA synthesis of miR-122 was carried out on C1000 PCR (Bio-Rad, Hercules, CA, USA). The presence of miR-122 was confirmed using Platinum SYBR Green qPCR Super Mix-UDG (Invitrogen) on a Step One Plus Real-Time PCR System (Applied Biosystems). U6 small nuclear RNA was used as the miRNA internal control. All primers designed using Primer Premier 5.0 (Premier BioSoft, Palo Alto, CA, USA) were synthesized by Invitrogen. Relative miRNA production was calculated using the $2^{-\Delta \Delta C t}$ method (Livak and Schmittgen, 2001), where Ct is the threshold cycle. All reactions were run in triplicate, and the results were normalized to U6 small nuclear RNA.

\section{Biochemical assay and pathological examination}

Serum alanine aminotransferase (ALT) and aspartate aminotransferase (AST) levels were determined using an automatic biochemical analyzer (Hitachi, Tokyo, Japan) according to manufacturer instructions. CuZn superoxide dismutase (SOD) and malondialdehyde (MDA) levels were determined using by Nanjing Jiancheng Bioengineering Institute (Nanjing, China). The protein of metallothionein (MT) and MRPS11 were detected using an enzyme-linked immunosorbent assay kit from the Shanghai Yanhui Biological Technology Institute (Shanghai, China). Formalinfixed samples were embedded in paraffin, sectioned, and stained with hematoxylin and eosin for microscopic examination.

\section{Statistical analysis}

The data are expressed as the mean \pm SD. Statistical differences between groups were determined using Student's $t$-tests. Multiple group comparisons were performed using analysis of variance in combination with a Tukey's or a Dunnett's post-hoc test. Differences with $\mathrm{P}<0.05$ were considered statistically significant. 


\section{RESULTS}

\section{Histopathology and biochemistry of mouse liver}

Histologic examinations were performed on liver specimens, the livers of the INH-treated mice exhibited inflammatory cell infiltration since the $3 \mathrm{D}$ time point $(\mathrm{P}<0.05)$ (Figure $1 \mathrm{~A}-\mathrm{H})$. Thus, an INH-induced liver injury model was established. Histopathological scores are shown in Table 1.
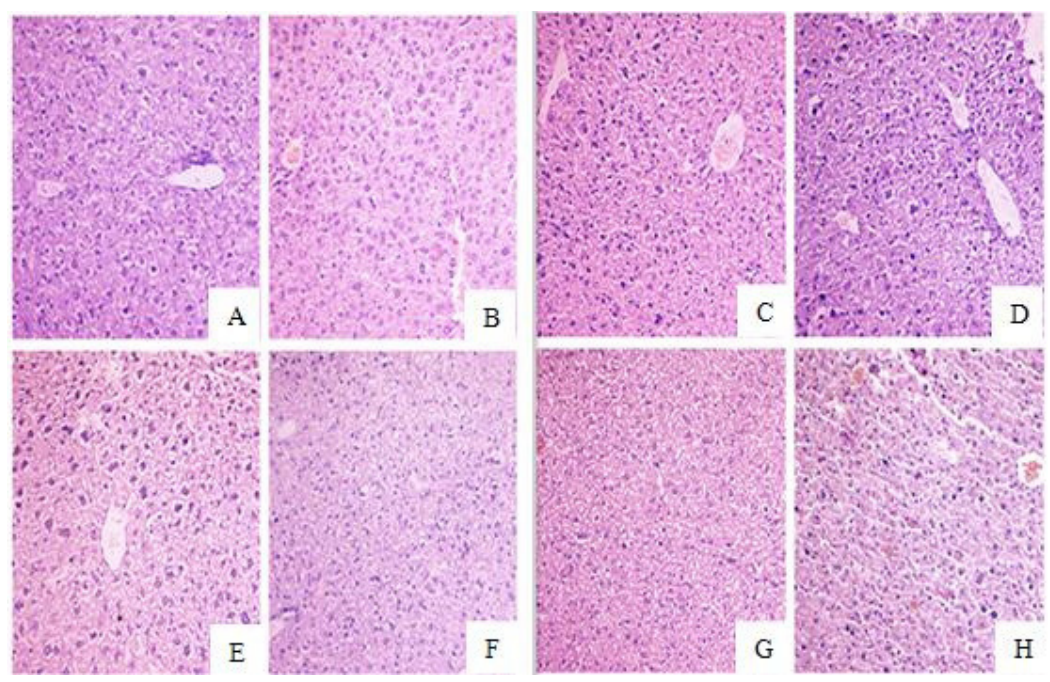

Figure 1. Effect of INH on liver histopathology in mice. Normal control group and model groups were administered 2 $\mathrm{mL}$ physiological saline and $90 \mathrm{mg} / \mathrm{kg} \mathrm{INH}$, respectively, orally once daily. Representative groups of hematoxylin-eosin staining of liver sections from normal control (A) and model groups of 1 day (B), 3 days (C), 5 days (D), 1 week (E), 2 weeks $(\mathbf{F}), 3$ weeks $(\mathbf{G})$, and 4 weeks $(\mathbf{H})$, respectively, time points (200X).

Table 1. Effect of INH on pathological changes of liver injury in mice.

\begin{tabular}{lc}
\hline Group & Score of liver injury \\
\hline Normal control & $0.00 \pm 0.00$ \\
Model 1 day & $0.10 \pm 0.31$ \\
Model 3 days & $0.70 \pm 0.67^{* \star}$ \\
Model 5 days & $1.00 \pm 0.47^{* \star}$ \\
Model 1 weeks & $1.50 \pm 1.08^{* \star}$ \\
Model 2 weeks & $1.90 \pm 0.99^{* \star}$ \\
Model 3 weeks & $3.50 \pm 0.71^{* \star}$ \\
Model 4 weeks & $4.10 \pm 0.99^{* *}$ \\
\hline
\end{tabular}

Histopathological score was calculated using the weighted number: liver cell congestion, hemorrhage $1 \mathrm{X}$, hepatocellular degeneration water $1 X$, inflammatory cell infiltration $1 X$, hepatocyte necrosis $3 X$. Mean $\pm S D$ are shown. Significantly different from control group $\left({ }^{*} \mathrm{P}<0.05,{ }^{* *} \mathrm{P}<0.01\right)$.

Serum ALT and AST levels were analyzed at different time points of INH-induced liver injury (Figure 2). The serum ALT and AST levels at 2 and 3 weeks after INH administration was significantly higher than those of the control group and considerably higher than those at the other time points. CuZnSOD and MDA levels in the liver tissues had significantly changed at 5 days. 
We found that that the significant changes in the general indicators of liver function (ALT and AST) and oxidative stress (CuZnSOD and MDA) $(P<0.05)$ occurred later than the histopathological changes caused by INH-induced liver injury.

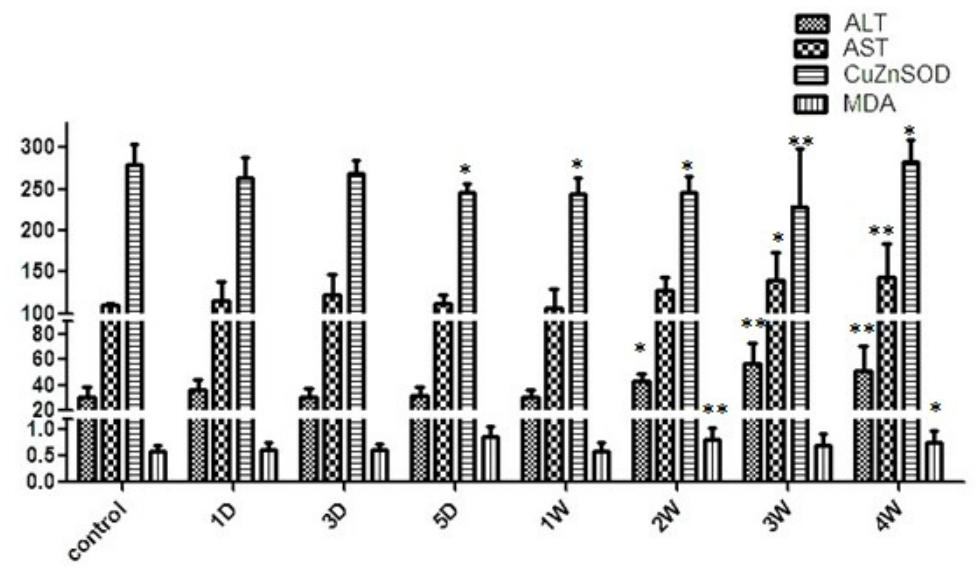

Figure 2. Effect of INH on biochemical parameters in mice. Compared with the control group, ALT at 2 weeks, AST at 3 weeks, CuZnSOD at 5 days, and MDA at 5 days were significantly changed. Mean \pm SD are shown. Significantly different from control group ( $\left.{ }^{*} P<0.05,{ }^{* *} P<0.01\right)$.

\section{Changes in tissue miR-122 in mice with INH-induced liver injury}

miR-122 levels in liver tissues were observed over time (Figure 3). The miR-122 level significantly decreased to $88.72 \pm 5.15 \%$ of the control at 3 days $(P<0.05)$. A decrease to 57.78 $\pm 1.56 \%$ of control levels was observed at 2 weeks. In addition, miR-122 levels increased after 2 weeks, but remained lower than control levels, suggesting that miR-122 expression decreased during INH-induced liver injury.

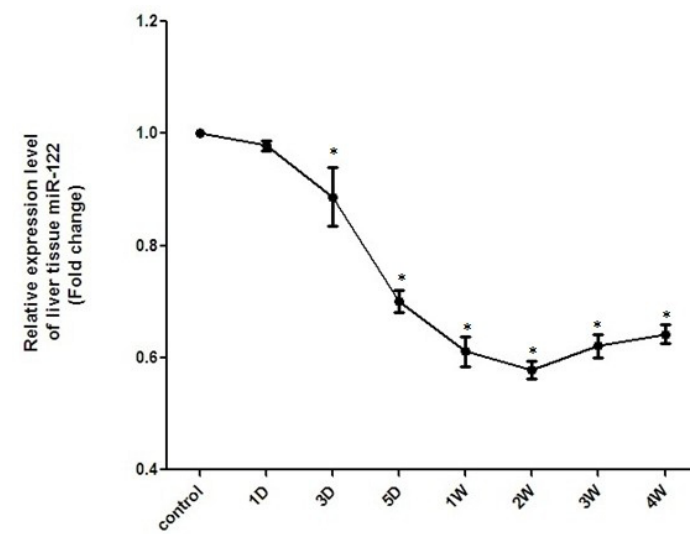

Figure 3. Effect of INH on relative miR-122 expression levels in mice. Abundance of miR-122 was measured by quantitative reverse transcription-polymerase chain reaction after normalization with U6 small nuclear RNA. Mean \pm $\mathrm{SD}$ are shown. Significantly different from control group ( ${ }^{*} \mathrm{P}<0.05$ ). Dates were 1 day, $97.89 \pm 0.88 \%$; 3 days, 88.72 $\pm 5.15 \%$; 5 days, $70.05 \pm 2.04 \%$; 1 week, $61.10 \pm 2.63 \%$; 2 weeks, $57.78 \pm 1.56 \%$; 3 weeks, $62.06 \pm 2.08 \%$; and 4 weeks, $64.19 \pm 1.73 \%$. 


\section{Changes in MT and MRPS11 protein levels in mice with INH-induced liver injury}

MT and MRPS11 protein levels were significantly altered after 3 days of INH treatment. Levels declined to lean at 2 weeks (908.68 $\pm 152.87,9.72 \pm 1.31)$ (Table 2).

\begin{tabular}{lcr}
\multicolumn{2}{c}{ Table 2. Effect of INH on liver tissue MT and MRPS11 levels in mice. } \\
\hline Group & MT (pg/mL) & MRPS11 (pg/mL) \\
\hline Normal control & $2230.93 \pm 669.06$ & $15.00 \pm 2.51$ \\
Model 1 day & $2559.84 \pm 1276.27$ & $14.66 \pm 2.65$ \\
Model 3 days & $1427.23 \pm 870.57^{*}$ & $14.40 \pm 3.26$ \\
Model 5 days & $2059.40 \pm 1023.77$ & $12.98 \pm 3.38^{*}$ \\
Model 1 week & $1411.49 \pm 526.41^{*}$ & $11.99 \pm 2.32^{*}$ \\
Model 2 weeks & $908.68 \pm 152.87^{*}$ & $9.72 \pm 1.31^{*}$ \\
Model 3 weeks & $1633.49 \pm 979.31$ & $10.98 \pm 2.84^{*}$ \\
Model 4 weeks & $1310.89 \pm 318.59^{*}$ & $11.78 \pm 0.67^{*}$ \\
\hline
\end{tabular}

Mean \pm SD are shown. *Significantly different from control group $(P<0.05)$.

\section{Correlations with biochemical parameters during INH liver injury}

We correlated the histopathological score with changes in biochemical parameters over time during INH-induced liver injury. The correlation coefficients of miR-122 ( $r=-0.741, P<0.01)$, ALT $(r=0.563, P<0.05)$, AST $(r=0.397, P<0.05)$, CuZnSOD $(r=-0.039, P>0.05)$, MDA $(r=$ $0.228, \mathrm{P}<0.05)$, MT $(r=-0.29, \mathrm{P}<0.01)$, and MRPS11 $(r=-0.392, \mathrm{P}<0.01)$ were determined. Additionally, the correlation between MRPS11 and CuZnSOD, MDA, and MT $(r=0.313, r=-0.250$, $r=-0.366 ; P<0.05)$ were determined.

\section{DISCUSSION}

ADLI is a highly organized process characterized by the progressive activation of precise pathways resulting specific biochemical and morphological alterations. In previous studies, it was demonstrated that oxidative stress takes part in liver injury (Zhu et al., 2014). We found that the activity of indices of oxidative stress (CuZnSOD, MDA) significantly changed during INH-induced liver injury at 7 time points (1,3, and 5 days and 1, 2, 3, and 4 weeks). Oxidative stress, initiated by ROS, is the collective pathophysiological mechanism of many hepatopathies. Excess ROS can lead to oxidative stress. The liver, however, which contains a large number of mitochondria that are the main source of ROS, is attacked (Qiu et al., 2014). CuZnSOD, an important oxidase, can scavenge superoxide anion radicals and reduce lipid peroxidation to play an important role in the body's oxidation and antioxidant balance. Adducts of MDA and protein may activate the immune response and cause liver damage. In our study, CuZnSOD and MDA varied at 5 days.

miRNA molecules have been investigated as potential markers of disease (Macha et al., 2014; Vilming Elgaaen et al., 2014; Wu et al., 2014), and the results of our recent study suggests miR-122 may be used as an ADLI marker. Increasing evidence suggests that the spatial and temporal specificities of miRNAs are important for tissue specification and cell lineage decisions (Fazi and Nervi, 2008). miRNAs regulate posttranscriptional gene expression by either RNA interference or inhibition of translational initiation and elongation (Bartel, 2004). miR-122, as a dominant liver-specific miRNA, may adjust the targets to affect liver cells (Ruoquan et al., 2014). We used a mouse INH-induced acute liver injury model and found that miR-122 decreased in liver 
tissue. miR-122 expression was more closely correlated with liver injury than were serum ALT and AST levels, indicating that miR-122 is a potential biomarker. The expression of miR-122 in tissue decreased in this study. Tissue miR-122 may originate in normal liver cells, and thus tissue miR-122 expression decreases when liver cells are necrotic. In this study, tissue miR-122 levels significantly changed at 3 days when during inflammatory cell infiltration.

MT, by scavenging free radicals, reduces liver toxicity caused by a variety of chemical substances, making it an endogenous cytoprotective agent. MRPS11, one of the targets of miR122 , is a mitochondrial ribosomal protein that influences mitochondrial to compose. The expression of tissue miR-122 decreases upon liver injury, resulting in decreased MRPS11 as well. In our study, the protein content of MT and MRPS11 decreased after treatment with INH. In this study, the indices of oxidative stress were altered during INH liver injury. At 5 days, the enzyme activity of CuZnSOD and MDA, was later than 3 days, the proteinable expression of MT and MRPS11.

Furthermore, the correlation coefficient between histopathological score and miR-122 revealed good correlation between CuZnSOD, MDA, and MT $(r=0.24, r=-0.311, r=0.415 ; P$ $<0.05)$. Thus, there might be a relationship between miR-122 levels and oxidative stress. The correlation coefficients between MRPS11 and CuZnSOD, MDA, and MT are 0.313, -0.250 , and $0.366(P<0.05)$. It may be that miR-122 affect to product mitochondrial; thus, oxidative stress would be starting, which is the main place of oxidative stress.

Tissue miR-122 is closely involved in INH-induced acute liver injury, and may be involved in oxidative stress by altering the levels of its target. Further studies involving various anti-tuberculosis drugs should be conducted to verify our results.

\section{Conflicts of interest}

The authors declare no conflict of interest.

\section{ACKNOWLEDGMENTS}

Research supported by the Science Foundation of the Key Laboratory of Tangshan (\#08150201A-1-8).

\section{REFERENCES}

Aghabozorg Afjeh SS and Ghaderian SM (2013). The role of microRNAs in cardiovascular disease. Int. J. Mol. Cell. Med. 2: 50-57.

Antoine DJ, Dear JW, Lewis PS, Platt V, et al. (2013). Mechanistic biomarkers provide early and sensitive detection of acetaminophen-induced acute liver injury at first presentation to hospital. Hepatology 58: 777-787.

Arataki K, Hayes CN, Akamatsu S, Akiyama R, et al. (2013). Circulating microRNA-22 correlates with microRNA-122 and represents viral replication and liver injury in patients with chronic hepatitis B. J. Med. Virol. 85: 789-798.

Bartel DP (2004). MicroRNAs: genomics, biogenesis, mechanism, and function. Cell 116: 281-297.

Burchard J, Zhang C, Liu AM, Poon RT, et al. (2010). microRNA-122 as a regulator of mitochondrial metabolic gene network in hepatocellular carcinoma. Mol. Syst. Biol. 6: 402.

Fazi F and Nervi C (2008). MicroRNA: basic mechanisms and transcriptional regulatory networks for cell fate determination. Cardiovasc. Res. 79: 553-561.

Fitzgerald KA and Caffrey DR (2014). Long noncoding RNAs in innate and adaptive immunity. Curr. Opin. Immunol. 26C: 140-146.

Henderson MC and Azorsa DO (2012). The genomic and proteomic content of cancer cell-derived exosomes. Front. Oncol. 2: 38 . 
Liu AM, Xu Z, Shek FH, Wong KF, et al. (2014). miR-122 targets pyruvate kinase M2 and affects metabolism of hepatocellular carcinoma. PLoS One 9: e86872.

Livak KJ and Schmittgen TD (2001). Analysis of relative gene expression data using real-time quantitative PCR and the 2(Delta Delta C(T)) Method. Methods 25: 402-408.

Macha MA, Seshacharyulu P, Krishn SR, Pai P, et al. (2014). MicroRNAs (miRNA) as biomarker(s) for prognosis and diagnosis of gastrointestinal (GI) cancers. Curr. Pharm. Des. 20: 5287-5297.

Qiu SL, Xiao ZC, Piao CM, Xian YL, et al. (2014). AMP-activated protein kinase $\alpha 2$ protects against liver injury from metastasized tumors via reduced glucose deprivation-induced oxidative stress. J. Biol. Chem. 289: 9449-9459.

Ruoquan Y, Wanpin N, Qiangsheng X, Guodong T, et al. (2014). Correlation between plasma miR-122 expression and liver injury induced by hepatectomy. J. Int. Med. Res. 42: 77-84.

Shahraki J, Motallebi A, Aghvami M and Pourahmad J (2013). Ichthyotoxic ochlodinium polykrikoides induces mitochondrial mediated oxidative stress and apoptosis in rat liver hepatocytes. Iran J. Pharm. Res. 12: 829-844.

Tsai WC, Hsu SD, Hsu CS, Lai TC, et al. (2012). MicroRNA-122 plays a critical role in liver homeostasis and hepatocarcinogenesis. J. Clin. Invest. 122: 2884-2897.

Vilming Elgaaen B, Olstad OK, Haug KB, Brusletto B, et al. (2014). Global miRNA expression analysis of serous and clear cell ovarian carcinomas identifies differentially expressed miRNAs including miR-200c-3p as a prognostic marker. BMC Cancer 14: 80

Wang B, Wang H and Yang Z (2012). MiR-122 inhibits cell proliferation and tumorigenesis of breast cancer by targeting IGF1R. PLoS One 7: e47053.

Wu HH, Lin WC and Tsai KW (2014). Advances in molecular biomarkers for gastric cancer: miRNAs as emerging novel cancer markers. Expert Rev. Mol. Med. 16: e1.

Zhang B, Sun S, Shen L, Zu X, et al. (2011). DNA methylation in the rat livers induced by low dosage isoniazid treatment. Environ. Toxicol. Pharmacol. 32: 486-490.

Zhang Y, Jia Y, Zheng R, Guo Y, et al. (2010). Plasma microRNA-122 as a biomarker for viral-, alcohol-, and chemical-related hepatic diseases. Clin. Chem. 56: 1830-1838.

Zhu X, Zhang F, Zhou L, Kong D, et al. (2014). Diallyl trisulfide attenuates carbon tetrachloride-caused liver injury and fibrogenesis and reduces hepatic oxidative stress in rats. Naunyn Schmiedebergs Arch. Pharmacol. 387: 445-455. 